\title{
Identification of Humans Using Robust Biometric Features
}

\author{
Byungjun Son, Jung-Ho Ahn, Ji-hyun Park, and Yillbyung Lee \\ Division of Computer and Information Engineering, Yonsei University \\ 134 Shinchon-dong, Seodaemoon-gu, Seoul 120-749, Korea \\ \{sonjun, jhpark, yblee\}@csai.yonsei.ac.kr \\ \{jungho\}@cs.yonsei.ac.kr
}

\begin{abstract}
The size of the feature set is normally large in a recognition system using biometric data, such as Iris, face, fingerprints etc. As dimensionality reduction is an important problem in pattern recognition, it is necessary to reduce the dimensionality of the feature space for efficient biometric identification. In this paper, we present one of the major discriminative learning methods, namely, Direct Linear Discriminant Analysis (DLDA). Also, we specifically apply the multiresolution decomposition of 2-D discrete wavelet transform to extract the robust feature set of low dimensionality from the acquired biometric data and to decrease the complexity of computation when using DLDA. This method of features extraction is well suited to describe the shape of the biometric data while allowing the algorithm to be translation and rotation invariant. The Support Vector Machines (SVM) approach for comparing the similarity between the similar and different biometric data can be assessed to have the feature's discriminative power. In the experiments, we have showed that that the proposed method for human iris and face gave a efficient way of representing iris and face patterns.
\end{abstract}

\section{Introduction}

The identification of humans for e.g. financial transactions, access control, or computer access has almost always been conducted by ID numbers, such as a PIN or a password. The main problem with those numbers is that, let aside the fact that they sometimes can be cracked quite easily, they can be stolen and used by and unauthorized person without detection. Biometric identification systems use personal features of the user itself to check the identity. If, for example, biometric features stored on a chip card are stolen, they cannot be used, because the imposter's biometric features do not match the features stored on the card. For this reason, the interest in biometric systems has risen very much lately. Many systems arise using eye, face, fingerprint, or voice features. All of them have different advantages and disadvantages. But they have the common disadvantage that the size of the feature set is normally large.

We discuss feature extraction strategies for a class of iris features with very high dimension. In a recognition system using the biometric features, one may 
try to use large feature set to enhance the recognition performance. However, the increase in the number of the biometric features has caused other problems. For example, the recognizer using higher dimension feature set requires more parameters to characterize the classifier and requires more storage. Thus, it will increase the complexity of computation and make its real-time implementation more difficult and costly. A larger amount of data is also needed for training. To avoid these problems, a number of dimensionality reduction algorithms have already been proposed to obtain compact feature set.

The feature extraction process needs to be effective so that salient features that can differentiate between various classes can be extracted [1]. Several methods like Principal Component Analysis (PCA) [2] [3], transform the input data so that the features are well separated and classification become easier. Linear transformations are extensively used because they are easy to compute and analytically tractable. Typically, these transformations involve projecting features from a high dimensional space to a lower dimensional space where they are well separated. Linear Discriminant Analysis (LDA) is one such discriminative technique based on Fisher's linear discriminant [4]. Linear Discriminant Analysis (LDA) and Principal Component Analysis (PCA) are two major methods used to extract new features [4].

In this paper, we deal with basic issues for iris and face recognition. Also, we use the wavelet and direct discriminant analysis [5] for high-dimensional data set of iris and face. Most of works on personal identification and verification by iris patterns have been done in 1990s, and recent noticeable studies among them include those of [6], [7] and [8]. Daugman and Wildes implemented a whole system for personal identification or verifications including the configuration of image acquisition device, whereas the Boles system only focused on the iris representation and matching algorithm without an image acquisition module.

This paper is organized as follows. Section 2 briefly describes the image preprocessing which mainly involves the quality check of an image and iris localization. In section 3, we overview a multilevel two-dimensional $2 \mathrm{D}$ discrete wavelet transform (DWT) to obtain the feature vector of an iris and face image with lower dimensionality and more robust features. Also, we describe the DLDA scheme to linearly transform the subimages of biometric data obtained by wavelet transform to new feature space with higher separability and lower dimensionality. The same operations of DWT and DLDA are performed in training as well as testing phases. Section 4 describes feature matching approach based on SVM. Experimental results and analysis will be stated in section 5, and finally the conclusions are given in section 6 .

\section{Image Preprocessing}

The images acquired from an image acquisition device always contain not only the appropriate images but also some inappropriate ones. Therefore, we need to check the quality of eye image to to determine whether the given images are appropriate for the subsequent processing or not and then to select the proper 


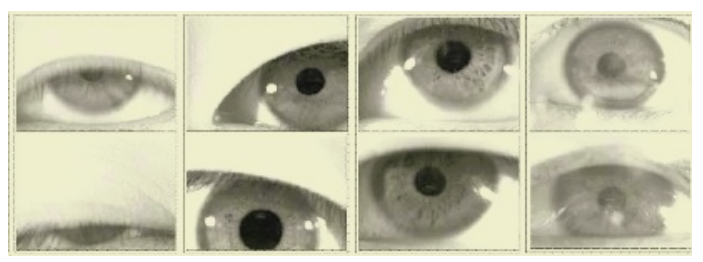

(a)

(b)

(c)

(d)

Fig. 1. Examples of images with bad quality: (a)the images with the blink (b)the images whose the pupil part is not located in the middle (c)the images obscured by eyelids or the shadow of the eyelids (d)the images with severe noises.

ones among them in real time. Some images ascertained as inappropriate ones are excluded from the next processing.

The images excluded from the subsequent processing include as follows; the images with the blink (Fig. 1(a)), the images whose the pupil part is not located in the middle and some parts of the iris area disappear (Fig. 1(b)), the images obscured by eyelids or the shadow of the eyelids (Fig. 1(c)), and the images with severe noises like Fig. 1(d). Fig. 1 shows the examples of images with bad quality.

An iris area can be localized from the eye image passed in the quality check step by separating the part of an image between the inner boundary and outer boundary. Fig. 2. shows the results of finding the inner boundary, the outer boundary and the collarette boundary in the eye image and the image of iris area, where is used in feature extraction, localized by using these boundaries.

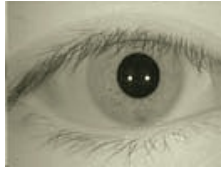

(a)

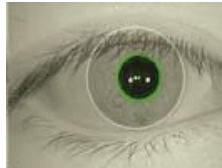

(b)

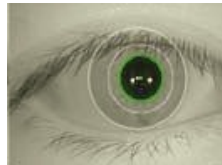

(c)

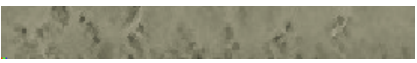

(d)

Fig. 2. (a) Original eye image (b)Image of the inner boundary and outer boundary (c)Image of the collarette boundary (d)localized iris image.

\section{Feature Extraction}

Most applications emphasize finding a feature set that produces efficient and implementable results. If the dimension of features defining a problem is too high, we must select a robust set of features from an initial set to provide appropriate 
representation. We also must design an appropriate classifier to the selected features set. We have chosen the DWT and DLDA approach to obtain a robust and lower dimensional set of features with high discriminating power.

\subsection{Wavelet Transform}

The hierarchical wavelet functions and its associated scaling functions are to decompose the original signal or image into different subbands. The decomposition process is recursively applied to the subbands to generate the next level of the hierarchy. The traditional pyramid-structured wavelet transform decomposes a signal into a set of frequency channels that have narrower bandwidths in the lower frequency region. The DWT was applied for texture classification and image compression because of its powerful capability for multiresolution decomposition analysis. The wavelet decomposition technique can be used to extract the intrinsic features for the recognition of persons by their biometric data. We employ the multilevel 2D Daubechies wavelet transform to extract the iris features. Using the wavelet transform, we decompose the image data into four subimages via the high-pass and low-pass filtering with respect to the column vectors and the row vectors of array pixels.

Figure 3 shows the process of pyramid-structured wavelet decomposition.

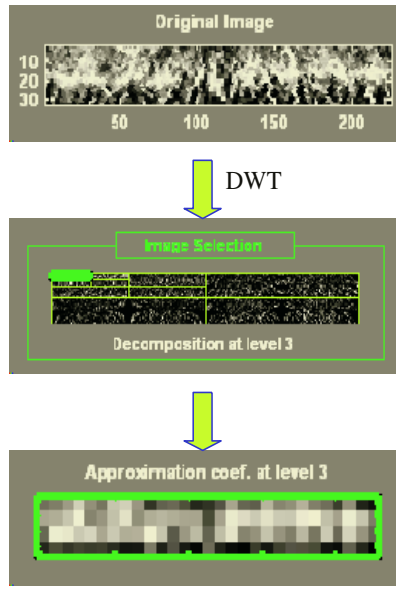

(a) Iris

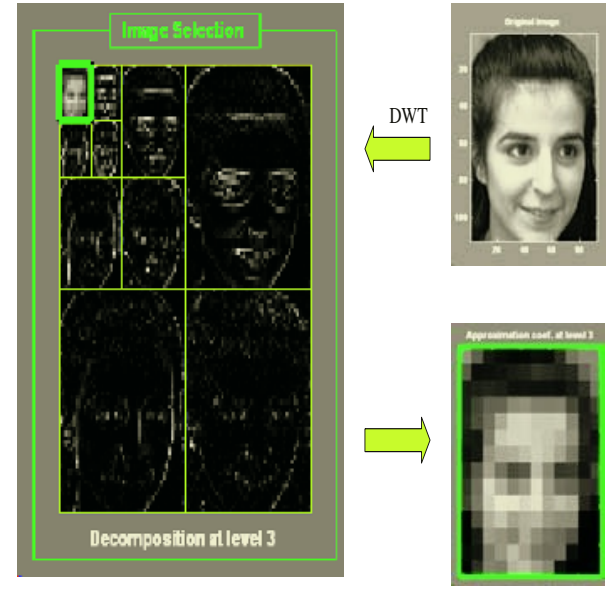

(b) Face

Fig. 3. Example of a 3-level wavelet transform of the iris and face images.

In this paper, we always select low frequency subimage for further decomposition. The three-level lowest frequency subimage is extracted as the feature vector. Generally, low frequency components represent the basic figure of an image, which is less sensitive to varying images. These components are the most informative subimages gearing with the highest discriminating power. The level 
of low frequency subimage chosen to extract the feature vector depends on size of the image. If the size is smaller then our localized iris image and ORL face, the one or two-level lowest frequency subimage might be have higher discriminating power.

\subsection{Direct Linear Discriminant Analysis}

After extraction of the iris and face feature vector by wavelet transform, the original iris vector $x$ of 7,200 dimensions is transformed to the feature vector $y$ of 116 dimensions. Also ORL face vector of 10,304 dimensions is reduced to 168 dimensions. To further reduce the feature dimensionality and enhance the class discrimination, we apply DLDA to convert the feature vector $y$ into a new discriminant vector $z$ with lower dimensions then the feature vector $y$.

Existing LDA methods first use PCA to project the data into lower dimensions, and then use LDA to project the data into an even lower dimension. The PCA step, however, can remove those components that are useful for discrimination. The key idea of DLDA method is to discard the null space of between-class scatter $S_{b}$ - which contains no useful information - rather than discarding the null space of $S_{w}$, which contains the most discriminative information [5]. Each scatter is given as follows:

$$
\begin{array}{rr}
S_{b}=\sum_{i=1}^{J} n_{i}\left(\mu_{i}-\mu\right)\left(\mu_{i}-\mu\right)^{T} & (n \times n) \\
S_{w}=\sum_{i=1}^{J} \sum_{x \in C_{i}}\left(x-\mu_{i}\right)\left(x-\mu_{i}\right)^{T} & (n \times n)
\end{array}
$$

where $n_{i}$ is the number of class i feature vectors, $\mu_{i}$ is the mean of class $i, \mu$ is the global mean, and $J$ is the number of classes.

The DLDA method is outlined below. We do not need to worry about the computational difficulty that both scatter matrices are too big to be held in memory because the dimensionality of input data is properly reduced by wavelet transform.

First, we diagonalize the $S_{b}$ matrix by finding a matrix $V$ such that

$$
V^{T} S_{b} V=D
$$

where the columns of $V$ are the eigenvectors of $S_{b}$ and $D$ is a diagonal matrix that contains the eigenvalues of $S_{b}$ in decreasing order. It is necessary to discard eigenvalues with 0 value and their eigenvectors, as projection directions with a total scatter of 0 do not carry any discriminative power at all [5].

Let $Y$ be the first $m$ columns of $V$ ( an $n \times m$ matrix, $n$ being the feature space dimensionality),

$$
Y^{T} S_{b} Y=D_{b} \quad(m \times m)
$$

where $D_{b}$ contains the $m$ non-zero eigenvalues of $S_{b}$ in decreasing order and the columns of $Y$ contain the corresponding eigenvectors. 
The next step is to let $Z=Y D^{1 / 2}$ such that $Z^{T} S_{b} Z=I$. Then we diagonalize the matrix $Z^{T} S_{w} Z$ such that

$$
U^{T}\left(Z^{T} S_{w} Z\right) U=D_{w}
$$

where $U^{T} U=I$. $D_{w}$ may contain zeros in its diagonal. We can sort the diagonal elements of $D_{w}$ and discard some eigenvalues in the high end, together with the corresponding eigenvectors.

We compute the LDA matrix as

$$
A=U^{T} Z^{T}
$$

Note that $A$ diagonalizes the numerator and denominator in Fisher's criterion.

Finally, we compute the transformation matrix(2) that takes an $n \times 1$ feature vector and transforms it to an $m \times 1$ feature vector.

$$
x_{\text {reduced }}=D_{b}^{-1 / 2} A x
$$

\section{SVM-Based Pattern Matching}

We only give here a brief presentation of the basic concepts needed. The reader is referred to [10] for a list of applications of SVMs. SVMs are based on structural risk minimization, which is the expectation of the test error for the trained machine. This risk is represented as $R(\alpha), \alpha$ being the parameters of the trained machine. Let $\beta$ be the number of training patterns and $0 \leq \gamma \leq 1$. Then, with probability $1-\gamma$ the following bound on the expected risk holds:

$$
R(\alpha) \leq R_{e m p}(\alpha)+\sqrt{\frac{h(\log (2 \beta / h)+1)-\log (\gamma / 4)}{\beta}}
$$

$R_{e m p}(\alpha)$ being the empirical risk, which is the mean error on the training set, and $\gamma$ is the VC dimension. SVMs try to minimize the second term of (4), for a fixed empirical risk.

For the linearly separable case, SVM provides the optimal hyperplane that separates the training patterns. The optimal hyperplane maximizes the sum of the distances to the closest positive and negative training patterns. This sum is called margin. In order to weight the cost of missclassification an additional parameter is introduced. For the non-linear case, the training patterns are mapped onto a high-dimensional space using a kernel function. In this space the decision boundary is linear. The most commonly used kernel functions are polynominals, gaussian, sigmoidal functions.

\section{Experimental Results}

Eye images were acquired through CCD camera with LED (Light-Emitting Diode) lamp around lens under indoor light. The size of eye images is pixels 
with 256 grey intensity values, and the size of normalized iris images is $32 \times 225$ pixels. Our data set consists of 1200 eye data acquired from 120 individuals (left and right eye). The images of the left and right eye were treated differently from the same person, because they have different patterns. Both males and females were among the data. The ages of all ranges from the early twenties to midthirties. In case of individuals with glasses, images are captured both removing their glasses and having their glasses; however, contact lenses remained place.

We also used face images from Olivetti-Oracle Research Lab(ORL) [11]. The ORL data set consists of 400 frontal faces: 10 tightly cropped images of 40 subjects with variations in poses, illuminations, facial expressions and accessories. The size of each image is $92 \times 112$ pixels, with 256 grey levels per pixel.

We randomly choose five images per person for training, the other five for testing. To reduce variation, each experiment is repeated at least 20 times. We applied LDA, DWT +LDA, DLDA, and DWT +DLDA to a training set. Also, we evaluated the recognition performances using nearest neighbor(NN) and SVM.

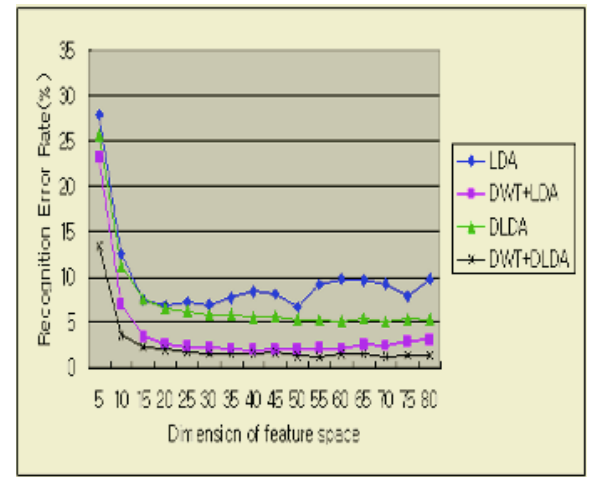

(a) Iris Results

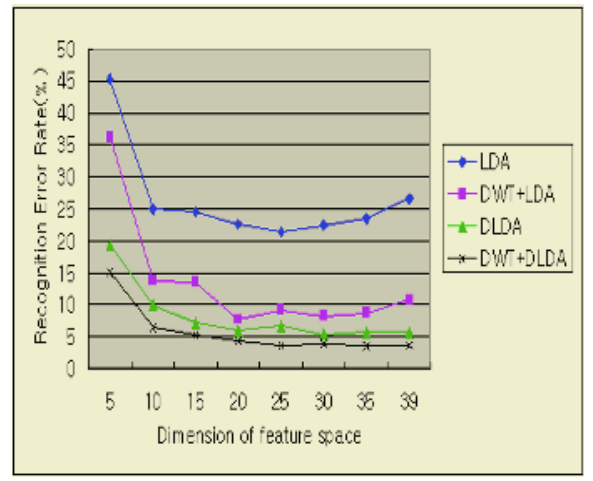

(b) Face Results

Fig. 4. Recognition Error Rate vs. Dimension of feature space.

Figure 4 shows the result of recognition error rate vs. dimension of feature space. The smallest recognition error rate of the LDA approach about the iris and face data is $6.7 \%$ and $21.42 \%$ with feature vector consisted of 20 and 25 components, respectively. The DLDA approach about the iris and face achieves $5.11 \%$ and 5.35 recognition error rate with feature vector consisted of 70 and 30 components, respectively. The DWT +LDA and DWT +DLDA approach about the iris achieve $1.96 \%$ and $1.24 \%$ with 40 and 55 features, respectively. About face achieve DWT +LDA and DWT +DLDA approach $7.75 \%$ and $3.57 \%$ with 20 and 35 features, respectively. the From Fig. 4, We can also see the DWT +DLDA method achieves the smallest recognition error rate. The recognition error rates of these DLDA methods are almost fixed after the number of features of iris and face reaches around 20. In addition, recognition rate of the DWT +DLDA approach about iris and face is $98.24 \%$ and $94.72 \%$ when the number 


\begin{tabular}{|c|c|c|}
\hline Dimension & DWT+DLDA+NN & DWT+DLDA+SVM \\
\hline 5 & 86.6 & 81.61 \\
\hline 10 & 96.41 & 95.25 \\
\hline 15 & 97.69 & 96.92 \\
\hline 20 & 97.97 & 98.03 \\
\hline 25 & 98.24 & 98.12 \\
\hline 30 & 98.4 & 98.52 \\
\hline 35 & 98.47 & 98.55 \\
\hline 40 & 98.44 & 98.61 \\
\hline 45 & 98.34 & 98.56 \\
\hline 50 & 98.69 & 99.21 \\
\hline 55 & 98.76 & 99.4 \\
\hline 60 & 98.51 & 99.13 \\
\hline 65 & 98.48 & 98.61 \\
\hline 70 & 98.76 & 99.35 \\
\hline 75 & 98.63 & 99.15 \\
\hline 80 & 98.66 & 99.18 \\
\hline
\end{tabular}

(a) Iris Results

\begin{tabular}{|c|c|c|}
\hline Dimension & $D W T+D L D A+N N$ & DWT+DLDA+SWM \\
\hline 5 & 84,9 & 86,13 \\
\hline 10 & 93,6 & 94,58 \\
\hline 15 & 94,72 & 95,7 \\
\hline 20 & 95,63 & 96,59 \\
\hline 25 & 96,38 & 96,82 \\
\hline 30 & 96,03 & 96,94 \\
\hline 35 & 96,43 & 97,53 \\
\hline 39 & 96,4 & 97,25 \\
\hline
\end{tabular}

(b) Face Results

Fig. 5. DWT $+\mathrm{DLDA}+\mathrm{NN}$ vs. DWT $+\mathrm{DLDA}+\mathrm{SVM}$.

of features is 25 and 15, respectively. It is higher than the best performance of the other methods and has lower dimension than others. This shows that the DWT+DLDA approach can achieve better performance although it uses smaller number of basis vectors than the others.

As compared in Fig. 5, we find that DWT +DLDA +SVM for gaussian kernel outperforms DWT +DLDA +NN. Such observations are almost consistent for different numbers of feature set. The best recognition rate of DWT+DLDA +SVM approach about the iris and face is $99.4 \%$ and 97.53 when the number of features is 55 and 35 , respectively.

\section{Conclusion}

In this paper, we have presented effective methods for the recognition of persons by their biometric features. We specifically uses the multiresolution decomposition of 2-D discrete wavelet transform for extracting the robust feature set of low dimensionality. In addition, the DLDA method is used to obtain the feature set with higher discriminative power and lower dimensionality. These methods of feature extraction well suit with iris and face recognition system while allowing the algorithm to be translation and rotation invariant.

We showed that the DWT+DLDA method outperformed the LDA, DWT $+\mathrm{LDA}$, and DLDA in terms of classification rate. For the complex data consisting of many classes in the problem of iris and face recognition, the DWT+DLDA method can be used for an alternative of LDA. Support vector machines also has the advantage of efficient testing, and good performance compared to other linear classifiers. For future works, it is necessary to conduct experiments on a large number of data so as to verify the efficiency and robustness of our approach. Other techniques for feature extraction and pattern matching can be handled from this point of view so as to propose the efficient methods for a reliable human recognition system. 


\section{Acknowledgements}

This work was supported in part by Brain Neuroinformatics Program sponsored by KMST.

\section{References}

1. H. Watanabe et al: "Discriminative Metric Design for Robust Pattern Recognition", IEEE Trans. On Signal Processing, vol. 45, PP. 2655-2662, 1997.

2. Ian T. Jollife: "Principal Component Analysis", Springer Verilag, New York, 1986.

3. Richard O. Duda, Peter E. Hart, David G. Stork: "Pattern Classification and Scene Analysis", Wiley Interscience, 2000.

4. W. L. Poston and Marchette, D. J.: "Recursive Dimensionality Reduction Using Fisher's Linear Discriminant," Pattern Recognition, 31(7):881-888, 1988.

5. Jie Yang, Hua Yu: "A Direct LDA Algorithm for High-Dimensional Data - with Application to Face Recognition," Pattern Recognition, 34(10):2067-2070, 2001.

6. John G. Daugman: "High confidence visual recognition of persons by a test of statistical indenpendence", IEEE Trans. On Pattern Analysis and Machine Intelligence, 15(11):1148-1161, January 1993.

7. R. P. Wiles: "Iris Recognition : An Emerging Biometric Technology," Proc. of the IEEE, 85(9):1348-1363 , 1997.

8. W. W. Boles, B. Boashash: "A Human Identification Technique Using Images of the Iris and Wavelet Transform," IEEETrans. of Signal Processing, 46(4):1185-1188, 1998.

9. Dimitrios Ioammou, Walter Huda, Andrew F. Laine: "Circle recognition through a 2D Hough Transform and radius histogramming," Image and Vision Computing, 7:15-26, 1999.

10. A. Tefas , C. Kotropoulos, and I. Pitas , "Using support vector machines to enhance the performance of elastic graph matching for frontal face authentication," IEEE Transactions on Pattern Analysis and Machine Intelligence, vol. 23, no. 7, 2001.

11. AT\&T Laboratories Cambridge. The ORL Database of Faces. http://www.cam-orl.co.uk/facedatabase.html 